\title{
Frequency regulation with thermostatic load participation in power networks
}

\author{
Andreas Kasis and Ioannis Lestas
}

\begin{abstract}
We consider the problem of controlling thermostatic loads such that ancillary services are provided to the power network within the secondary frequency control timeframe. This problem has been widely studied in the literature, where stochastic control schemes have been proposed to avoid the possibility of load synchronization, which induces persistent frequency oscillations. However, stochastic schemes introduce delays in the response of thermostatic loads that may limit their ability to provide support at urgencies. In this paper, we present a deterministic control mechanism for thermostatic loads such that those switch when prescribed frequency thresholds are exceeded in order to provide ancillary services to the power network. For the considered scheme, we propose appropriate conditions for the design of the frequency thresholds that bound the coupling between frequency and thermostatic load dynamics, so as to avoid synchronization phenomena. In particular, we show that as the number of loads tends to infinity, there exist arbitrarily long time intervals where the frequency deviations are arbitrarily small.
\end{abstract}

\section{INTRODUCTION}

Motivation and literature review: A significant growth in the penetration of renewable sources of generation in power networks is expected in the following years [1], [2], driven by environmental concerns. This will result in increasingly intermittent generation, endangering power quality and potentially the stability of the power network. Controllable loads are considered to be a way to counterbalance intermittent generation, due to their ability to provide fast response at urgencies by adapting their demand accordingly. The use of loads as ancillary services, in conjunction with a large penetration of renewable sources of generation will significantly increase the number of active devices in the network making its electromechanical behavior difficult to predict and encouraging the analytical study of its behaviour. Along these lines, various research studies in recent years have considered controllable demand as a means of providing support to primary [3], [4], [5], [6], [7], and secondary [8], [9], [10], [11], [12], [13], frequency control mechanisms, where the objective is to ensure that generation and demand are balanced and that the frequency converges to its nominal value $(50 \mathrm{~Hz}$ or $60 \mathrm{~Hz})$ respectively.

Thermostatic loads comprise a significant portion of the total demand. A recent survey in the EU [14] showed that thermostatic loads exceeded $80 \%$ and $40 \%$ of the total consumption in households with and without electric heating respectively. Hence, an analytic study of the thermostatic

This work was supported by ERC starting grant 679774 .

Andreas Kasis and Ioannis Lestas are with the Department of Engineering, University of Cambridge, Trumpington Street, Cambridge, CB2 1PZ, United Kingdom; e-mails: ak647@cam.ac.uk, ic120@cam.ac.uk effects on load behavior is of great importance if those are to provide ancillary services to the power network.

The use of thermostatic loads as a means for providing support to frequency control has been considered in [15], where the authors suggested temperature thresholds in thermostatic loads to be linearly dependent on frequency and demonstrated with simulations that this resulted in improved performance. However, it was demonstrated in [16] that such control scheme could potentially result to load synchronization, i.e. many loads simultaneously switching and causing large oscillations in the power network. As a remedy to this problem, the authors proposed a stochastic control scheme which ensured that load would not synchronize. Various other studies [17], [18], [19], considered similar problems by relying on stochastic approaches. However, stochastic approaches induce time delays in the response of thermostatic loads at fast frequency fluctuations, which may limit their applicability. The latter, motivates the study of deterministic schemes for the control of thermostatic loads, such that a faster response is allowed at urgencies.

Contribution: This study considers a deterministic approach for the control of thermostatic loads, such that ancillary services are provided at urgencies. Our main analytic results concern the case when the number of loads tends to infinity, a condition justified by the large number of thermostatic appliances in power networks, e.g. around 40 million refrigerators are estimated to operate in the U.K. [17].

We first consider a conventional scheme for thermostatic loads where they do not contribute to frequency control, and show that when their number tends to infinity, the variance of their aggregate sum tends to zero.

We then consider a control scheme for thermostatic loads, such that loads switch when certain frequency thresholds are exceeded in order to support existing secondary frequency control schemes. The considered scheme ensures that load temperatures will not exceed their respective bounds, and hence that user comfort levels will not be affected. Furthermore, the proposed scheme, being deterministic, enables the instant switch of loads at urgencies, hence allowing faster frequency response compared to stochastic approaches. For the considered scheme, we provide design conditions for the frequency thresholds that bound the coupling between the frequency and the load dynamics so as to avoid load synchronization. In particular, we analytically show that when the number of loads tends to infinity, the frequency deviations will be arbitrarily small for arbitrarily long time intervals.

Paper structure: In Section II we present some basic notation used in the paper and in Section III the considered 
power system. In Section IV we consider a conventional model for thermostatic loads and study its properties in terms of the aggregate mean and variance. In Section V, we present our proposed scheme for frequency control using thermostatic loads and state our main results regarding the performance of the power system. Finally, conclusions are drawn in Section VI. The proofs of the results have been omitted due to page constraints and will be included in an extended version of this work.

\section{NOTATION}

Real numbers are denoted by $\mathbb{R}$ and $\mathbb{N}$, and the set of $n$ dimensional vectors with real entries is denoted by $\mathbb{R}^{n}$. Furthermore, we define the sets of integers and positive rational and positive real numbers by $\mathbb{Z}, \mathbb{Q}_{+}$and $\mathbb{R}_{+}$respectively. The set of natural numbers and the set of natural numbers including zero are denoted by $\mathbb{N}$ and $\mathbb{N}_{0}$ respectively. The cardinality of a set $S$ is denoted by $|S|$. For $a \in \mathbb{R}, b \in$ $\mathbb{R} \backslash\{0\}$ the modulo of $a$ to $b$ is denoted by $[a]_{b}^{+}$and defined as $[a]_{b}^{+}=a-b\left\lfloor\frac{a}{b}\right\rfloor$, where for $x \in \mathbb{R},\lfloor x\rfloor=\sup \{m \in \mathbb{Z}$ : $m \leq x\}$. The average of a real valued time signal $x(t)$ with respect to time is defined as $\mathbb{E}_{t}(x(t))=\lim _{\tau \rightarrow \infty} \frac{1}{\tau} \int_{0}^{\tau} x(t) d t$ and its variance as $\mathbb{V}_{t}(x(t))=\mathbb{E}_{t}\left((x(t))^{2}\right)-\left[\mathbb{E}_{t}(x(t))\right]^{2}$. Note that for convenience in presentation we shall use $\mathbb{E}$ and $\mathbb{V}$ (without the subscript $t$ ) to denote the mean and variance with respect to time.

\section{POWER SYSTEM MODEL}

We use the swing equation to describe the rate of change of the frequency of the power system (e.g. [20]). In particular, we consider the following assumptions on our studied model: 1) Bus voltage magnitudes satisfy $|V|=1$ p.u. for all buses. 2) Lines are lossless and characterized by their susceptances. 3) Reactive power flows do not affect bus voltage phase angles and frequencies.

4) Frequencies between buses are synchronized.

The first three conditions have been widely used in the literature for the study of frequency control schemes in power networks [10], [13]. The fourth assumption is justified from the relatively small deviations between bus frequencies, which allows the study of power system characteristics using a single frequency (see also [21], [22]). This motivates the following system dynamics,

$$
M \dot{\omega}=-p^{L}+p^{M}-D \omega-\sum_{j \in N} d_{j}^{c} .
$$

In system (1) the time-dependent variables $p^{M}, d_{j}^{c}$ and $\omega$ represent, respectively, the mechanical power injection, the $j$ th thermostatic load and the deviation from the nominal value ${ }^{1}$ of frequency. Furthermore, we let $N:=\{1,2 \ldots,|N|\}$ be the set of thermostatic loads. The constants $M>0$ and $D>0$ denote the generator inertia and damping coefficient respectively. We study the response of system (1) at a step change in the uncontrollable demand

\footnotetext{
${ }^{1} \mathrm{~A}$ nominal value is defined as an equilibrium of (1) with frequency equal to $50 \mathrm{~Hz}$ (or $60 \mathrm{~Hz}$ ).
}

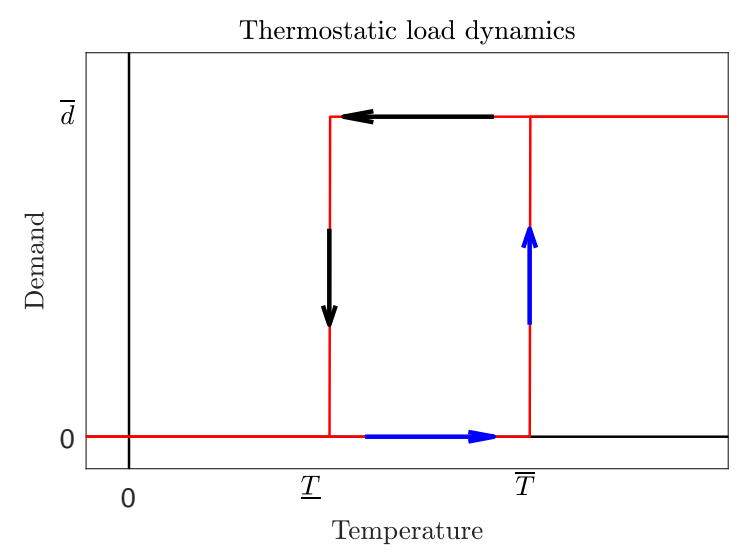

Fig. 1: Thermostatic loads scheme described by (3).

$p^{L}$. Furthermore, we shall consider generation dynamics described by

$$
\dot{p}^{M}=-K \omega,
$$

where $K>0$ is the integral gain. Note that the simplicity in the generation dynamics (2) is in order to facilitate the analysis of the paper and keep its focus on thermostatic loads, which have a non-trivial hybrid and periodic behaviour as it will be discussed within the paper. More advanced generation models will be considered as part of future work.

\section{THERMOSTATIC LOADS}

In this section we consider the conventional model for thermostatic loads and study its properties. The analysis in this section deduces important properties of thermostatic load behavior, which are used to obtain the main results of this paper. Thermostatic load dynamics are conventionally (e.g [16], [23]) described by

$$
d_{j}^{c}=\bar{d}_{j} \sigma_{j}, \quad \sigma_{j}\left(t^{+}\right)= \begin{cases}1, & T_{j} \geq \bar{T}_{j}, \\ 0, & T_{j} \leq \underline{T}_{j}, \\ \sigma_{j}(t), & \underline{T}_{j} \leq T_{j} \leq \bar{T}_{j},\end{cases}
$$

where $j \in N$ and $t^{+}=\lim _{\epsilon \rightarrow 0}(t+\epsilon)$. In (3), the timedependent variables $d_{j}^{c}$ and $\sigma_{j}$ denote the demand and switch state of the $j$ th load respectively. The constants $\bar{d}_{j}, \underline{T}_{j}$ and $\bar{T}_{j}$ denote the load magnitude and lower and upper temperature thresholds for load $j$ respectively and satisfy $\bar{d}_{j} \in \mathbb{R}_{+}$and $\bar{T}_{j}>\underline{T}_{j}>0, j \in N$. The hysteresis scheme in (3) is depicted in Figure 1.

Furthermore, the temperature dynamics satisfy

$$
\dot{T}_{j}=-k_{j}\left(T_{j}-\hat{T}_{j}+\lambda_{j} d_{j}^{c}\right), j \in N,
$$

where $T_{j}, k_{j}, \lambda_{j}>0$ denote the time-dependent temperature, thermal insulation coefficient and coefficient of performance of load $j$ respectively. Furthermore, $\hat{T}_{j}$ denotes the ambient temperature of load $j$ that is assumed constant. Moreover, it is assumed that $\hat{T}_{j}-\lambda_{j} \bar{d}_{j}<\underline{T}_{j}$, such that (3), (4), has no equilibria, as is the case in practise. 


\section{A. Period and ON-time of thermostatic loads}

The period $\pi_{j}$ of thermal load $j$, described by (3), (4), is defined as the time required for load $j$ to switch twice, i.e. the time between two consecutive switches to the ON (or equivalently $\mathrm{OFF}$ ) state. In the following definition, we let $t_{j, i}$ be the time where the $i$ th switch of load $j$, described by (3), (4), occurs.

Definition 1: The period of load $j$ is defined as $\pi_{j}=$ $t_{j, i+2}-t_{j, i}$, for any $i \geq 1$.

It should be clear that for any $j \in N$, it holds that $t_{j, i+2}-$ $t_{j, i}=t_{j, k+2}-t_{j, k}$, for all $i, k \in \mathbb{N}$. Note that the time lengths that load $j$ remains switched $\mathrm{ON}$ and OFF within each period are respectively given by

$$
\begin{gathered}
\pi_{j}^{O N}=\frac{1}{k_{j}} \ln \left(\frac{\bar{T}_{j}+\lambda_{j} \bar{d}_{j}-\hat{T}_{j}}{\underline{T}_{j}+\lambda_{j} \bar{d}_{j}-\hat{T}_{j}}\right), j \in N, \\
\pi_{j}^{O F F}=\frac{1}{k_{j}} \ln \left(\frac{\hat{T}_{j}-\underline{T}_{j}}{\hat{T}_{j}-\bar{T}_{j}}\right), j \in N,
\end{gathered}
$$

and that it trivially follows that $\pi_{j}=\pi_{j}^{O N}+\pi_{j}^{O F F}$. Furthermore, we let $\alpha_{j}=\frac{\pi_{j}^{O N}}{\pi_{j}}$ be the ratio of time each load stays switched ON within each period. Moreover, we define the period ratio between loads $i$ and $j$ as $\rho_{i j}=\frac{\pi_{i}}{\pi_{j}}$. We shall use $d_{j}^{c, *}=\alpha_{j} \bar{d}_{j}$ to denote the average value of $d_{j}^{c}$ when its dynamics are described by (3), (4). Finally, we let

$$
d^{s}=\sum_{j \in N} d_{j}^{c}, \quad \Gamma=\sum_{j \in N} \bar{d}_{j},
$$

be the aggregate sum and aggregate magnitude of thermostatic loads, where $\Gamma \in \mathbb{R}_{+}$.

\section{B. Expectation and variance analysis}

In this section we consider the behavior of the aggregation of thermostatic loads in terms of mean and variance. In particular, we study how these two quantities are influenced when the number of loads tends to infinity, assuming always a constant aggregate sum.

An important assumption for the following analysis is that all period ratios lie in the set $\mathbb{R}_{+} / \mathbb{Q}_{+}$. Note that $\mathbb{Q}_{+}$is of measure zero and hence the condition $\rho_{i j} \in \mathbb{R}_{+} / \mathbb{Q}_{+}$is unlikely to be violated in practise. The assumption is stated below.

Assumption 1: All loads $i, j \in N$ described by (3), (4), satisfy $\rho_{i j} \in \mathbb{R}_{+} / \mathbb{Q}_{+}$.

Assumption 1 is a technical condition for Theorem 1 below showing that the variance of the aggregation of loads is zero for any initial condition. Assumption 1 excludes cases such as when two loads have identical periods, which makes the trajectory of the aggregation of any two loads periodic and depended on the initial conditions. The latter is true for all cases where $\rho_{i j} \in \mathbb{Q}_{+}$, which are hence excluded.

The following theorem states that the variance of the aggregation of thermostatic loads tends to zero as their number tends to infinity.

Theorem 1: Consider thermostatic loads described by (3), (4), with $\bar{d}_{j}=\frac{\Gamma}{|N|}$ and let Assumption 1 hold. Then, $\lim _{|N| \rightarrow \infty} \mathbb{V}\left(d^{s}\right)=0$.
Theorem 1 demonstrates that as the number of loads tends to infinity, the variance of their aggregation tends to zero. This suggests that as the number of loads described by (3), (4), in the power network becomes large, then an almost flat load aggregation should be expected, a desired feature to obtain a smooth frequency response. Note that Theorem 1, as well as many of the results that follow, are stated for the case where $\bar{d}_{j}=\frac{\Gamma}{|N|}, j \in N$, which suggests a constant aggregate sum $\Gamma$ and loads of identical magnitude. Although the former is essential for the presented analysis, the assumption that all load magnitudes are identical is made only for simplicity and could potentially be relaxed, as part of future work.

\section{FREQUENCY CONTROL OF THERMOSTATIC LOADS}

In this section we consider the use of frequency feedback for the control of thermostatic loads. In particular, we present a frequency control scheme for thermostatic loads and propose appropriate conditions for its design. For the proposed scheme, we show that, as the number of loads tends to infinity, then no synchronization phenomena occur and that there exist arbitrarily long time intervals where frequency deviations are arbitrarily small.

\section{A. Frequency control scheme for thermostatic loads}

In this section we present a novel scheme to control thermostatic loads such that ancillary services are provided at urgencies, i.e. when frequency deviations exceed particular thresholds. The scheme, depicted on Figure 2, is described below

$$
\sigma_{j}\left(t^{+}\right)=\left\{\begin{array}{c}
d_{j}^{c}=\bar{d}_{j} \sigma_{j}, \\
0, \quad\left\{\begin{array}{l}
T_{j} \geq \bar{T}_{j}, \\
\omega \geq \omega_{j}^{1} \text { and } \underline{T}_{j}+\epsilon_{j} \leq T_{j} \leq \bar{T}_{j}-\epsilon_{j}, \\
T_{j} \leq \underline{T}_{j},
\end{array}\right. \\
\sigma_{j}(t), \quad\left\{\begin{array}{l}
|\omega| \leq \omega_{j}^{1} \text { and } \underline{T}_{j}+\epsilon_{j} \leq T_{j} \leq \bar{T}_{j}-\epsilon_{j}, \\
T_{j} \in\left[\underline{T}_{j}, \underline{T}_{j}+\epsilon_{j}\right] \cup\left[\bar{T}_{j}, \bar{T}_{j}-\epsilon_{j}\right] .
\end{array}\right.
\end{array}\right.
$$

where $\omega_{j}^{1}>0$ are frequency thresholds and $0<\epsilon_{j}<\left(\bar{T}_{j}-\right.$ $\left.\underline{T}_{j}\right) / 2, j \in N$. Note that, $\epsilon_{j}$ in (7) serves to ensure than no Zeno behaviour should be expected as a result of the coupling between the frequency and thermostatic load dynamics. The latter is analytically shown in Lemma 1 below.

The scheme in (7) responds to frequency deviations by switching when prescribed frequency thresholds are exceeded thus providing ancillary services to the power network. Furthermore, when the frequency deviation does not reach the corresponding frequency thresholds, then the scheme in (7) reduces to (3). Note that, according to (7), temperature will always be within its respective thresholds and hence users comfort levels will not be affected.

For the rest of the manuscript, we let $S(\omega)=\left\{j: \omega_{j}^{1} \leq\right.$ $\omega\}$ be the set of loads with respective upper frequency thresholds below $\omega$. Moreover, for any set $S \subseteq N$, we let $\omega_{m}(S)=$ 


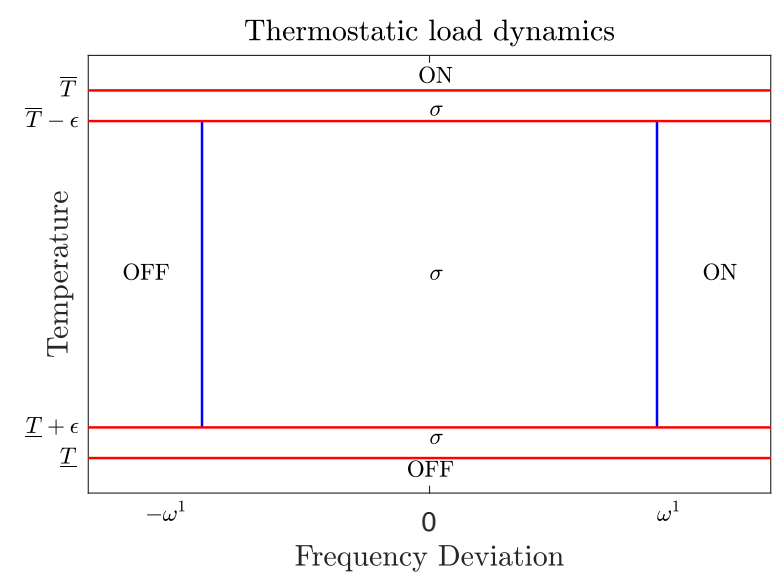

Fig. 2: Thermostatic loads scheme described by (7).

$\min _{j \in S} \omega_{j}^{1}, d_{S}^{s}(t)=\sum_{j \in S} d_{j}^{c}(t)$ and $d_{S}^{s, *}=\sum_{j \in S} \alpha_{j} \bar{d}_{j}$ Furthermore, we define the following parameters

$$
L=\left\{\begin{array}{l}
\frac{D}{M K}, \text { if } D^{2} \geq 4 M K \\
\left.2\left(\frac{1}{D^{2}}+\frac{1}{4 M K-D^{2}}\right)^{\frac{1}{2}}\right), \text { otherwise. }
\end{array}\right.
$$

$\hat{L}=\left\{\begin{array}{l}\frac{1}{M \hat{\omega}}\left(d^{\frac{\hat{\omega}-\beta}{2 \hat{\omega}}}-d^{-\frac{\hat{\omega}+\beta}{2 \hat{\omega}}}\right) \text { if } D^{2} \geq 4 M K \\ \frac{2}{M \bar{\omega} c}\left(\frac{\beta}{\bar{\omega} c}+\left(e^{-\frac{\beta}{\omega}\left(\phi+\frac{\pi}{2}\right)}\right) /\left(1-e^{-\frac{\beta}{\omega} \pi}\right)\right), \text { otherwise, }\end{array}\right.$

where $\beta=\frac{D}{2 M}, \hat{\omega}=\frac{\sqrt{D^{2}-4 M K}}{2 M}, \bar{\omega}=j \hat{\omega}, d=\frac{\beta+\hat{\omega}}{\beta-\hat{\omega}}$, $c=\left(1+\frac{\beta^{2}}{\bar{\omega}^{2}}\right)^{\frac{1}{2}}$ and $\phi \in\left\{\tan ^{-1}\left(\frac{\beta}{\bar{\omega}}\right)\right\} \cap\left(0, \frac{\pi}{2}\right)$. Note that it is trivial to show that $L, \hat{L} \in \mathbb{R}_{+}$when $M, K, D \in \mathbb{R}_{+}$.

The following condition is imposed for the design of frequency thresholds.

Design condition 1: The frequency thresholds $\omega_{j}^{1}$ are chosen such that, for all $\omega \in \mathbb{R}$ and some $\delta>0$, at least one of the following holds for $L, \hat{L}$ defined by (8)

(i) $\sum_{j \in S(|\omega|)} \bar{d}_{j} \leq \max (\mathcal{K}(|\omega|-\delta), 0)$, where $\mathcal{K}=L^{-1}$,

(ii) $\sum_{j \in S(|\omega|)} \bar{d}_{j} \leq \max (\hat{\mathcal{K}}(|\omega|-\delta), 0)$, where $\hat{\mathcal{K}}=\hat{L}^{-1}$.

It should be clear that $\delta$ in Design condition 1 satisfies $\delta \in\left(0, \omega_{m}(N)\right)$ by definition, since $\omega_{j}^{1}<\delta$ for some $j \in N$ would imply that Design condition 1 does not hold. Design condition 1 is important to deduce that no synchronization occurs between thermostatic loads when the scheme (7) is considered. The respective values of $L$ and $\hat{L}$ consist an upper bound and the exact value of the 1-norm of the system (1), (2), with input $d^{s}$ and output $\omega$, which is relevant in the analysis below. This suggests that part (ii) is a relaxation of part (i) allowing for a less conservative bound on allowable loads. This bound becomes significantly better at particular parametric realizations of (1), (2), e.g. when $4 M K>D^{2}$ and $D^{2} \rightarrow 4 M K$. On the other hand, part (i) provides an intuitive and easy to implement condition that is satisfactory for many practical cases.

\section{B. Hybrid system description}

The behavior of system (1), (2), (4), (7), can be described by the states $z=(x, \sigma)$, where $x=\left(\omega, p^{M}, T\right) \in \mathbb{R}^{n}, n=$ $|N|+2$, is the continuous state, and $\sigma \in P^{|N|}$ the discrete state, where $P=\{0,1\}$. Moreover, let $\Lambda=\mathbb{R}^{n} \times P^{|N|}$ be the space where the system states evolve. The continuous dynamics of the system (1), (2), (4), (7), are described by

$$
\begin{gathered}
M \dot{\omega}=-p^{L}+p^{M}-D \omega-\sum_{j \in N} \bar{d}_{j} \sigma_{j} \\
\dot{p}^{M}=-K \omega, \\
\dot{T}_{j}=-k_{j}\left(T_{j}-\hat{T}_{j}+\lambda_{j} d_{j}^{c}\right), j \in N, \\
\dot{\sigma}_{j}=0, j \in N,
\end{gathered}
$$

which is valid when $z$ belongs to the set $C$ given by

$$
C=\left\{z \in \Lambda: \sigma_{j} \in \mathcal{I}_{j}\left(T_{j}, \omega\right), \forall j \in N\right\}
$$

where

$$
\mathcal{I}_{j}\left(T_{j}, \omega\right)=\left\{\begin{array}{l}
\{1\},\left\{\begin{array}{l}
T_{j}>\bar{T}_{j}, \\
\omega>\omega_{j}^{1} \text { and } \underline{T}_{j}+\epsilon_{j}<T_{j}<\bar{T}_{j}-\epsilon_{j},
\end{array}\right. \\
\{0\},\left\{\begin{array}{l}
T_{j}<\underline{T}_{j}, \\
\omega<-\omega_{j}^{1} \text { and } \underline{T}_{j}+\epsilon_{j}<T_{j}<\bar{T}_{j}-\epsilon_{j},
\end{array}\right. \\
\{0,1\},\left\{\begin{array}{l}
|\omega| \leq \omega_{j}^{1} \text { and } \underline{T}_{j} \leq T_{j} \leq \bar{T}_{j}, \\
T_{j} \in\left[\underline{T}_{j}, \underline{T}_{j}+\epsilon_{j}\right] \cup\left[\bar{T}_{j}, \bar{T}_{j}-\epsilon_{j}\right] .
\end{array}\right.
\end{array}\right.
$$

Alternatively, when $z$ belongs to the set $D=(\Lambda \backslash C) \cup \underline{D}$ where $\underline{D}=\left\{z \in \Lambda: \sigma_{j} \in \mathcal{I}_{j}^{D}\left(T_{j}, \omega\right), \forall j \in N\right\}$, and

$$
\mathcal{I}_{j}^{D}\left(T_{j}, \omega\right)=\left\{\begin{aligned}
\{1\},\left\{\begin{array}{l}
T_{j}=\underline{T}_{j}, \\
\omega=-\omega_{j}^{1} \text { and } T_{j} \in\left[\underline{T}_{j}+\epsilon_{j}, \bar{T}_{j}-\epsilon_{j}\right], \\
\omega \leq-\omega_{j}^{1} \text { and } T_{j} \in\left\{\underline{T}_{j}+\epsilon_{j}, \bar{T}_{j}-\epsilon_{j}\right\},
\end{array}\right. \\
\{0\},\left\{\begin{array}{l}
T_{j}=\bar{T}_{j}, \\
\omega=\omega_{j}^{1} \text { and } T_{j} \in\left[\underline{T}_{j}+\epsilon_{j}, \bar{T}_{j}-\epsilon_{j}\right], \\
\omega \geq \omega_{j}^{1} \text { and } T_{j} \in\left\{\underline{T}_{j}+\epsilon_{j}, \bar{T}_{j}-\epsilon_{j}\right\},
\end{array}\right.
\end{aligned}\right.
$$

then its components follow the discrete update described below

$x^{+}=x, \sigma_{j}\left(t^{+}\right)=\left\{\begin{array}{l}1,\left\{\begin{array}{l}T_{j} \geq \bar{T}_{j}, \\ \omega \geq \omega_{j}^{1} \text { and } T_{j} \in\left[\underline{T}_{j}+\epsilon_{j}, \bar{T}_{j}-\epsilon_{j}\right],\end{array}\right. \\ 0,\left\{\begin{array}{l}T_{j} \leq \underline{T}_{j}, \\ \omega \leq-\omega_{j}^{1} \text { and } T_{j} \in\left[\underline{T}_{j}+\epsilon_{j}, \bar{T}_{j}-\epsilon_{j}\right] .\end{array}\right.\end{array}\right.$

We can now provide the following compact representation for the hybrid system (1), (2), (4), (7),

$$
\begin{gathered}
\dot{z}=f(z), z \in C, \\
z^{+}=g(z), z \in D,
\end{gathered}
$$

where $f(z): C \rightarrow \Lambda$ and $g(z): D \rightarrow C$ are described by (9) and (11) respectively. Note that $z^{+}=g(z)$ represents a discrete dynamical system where $z^{+}$indicates that the next value of the state $z$ is given as a function of its current value through $g(z)$. Moreover, notice that $C \cup D=\Lambda$. 


\section{Analysis of solutions}

In this section we consider the solutions of (12) and show their existence and that no Zeno behavior occurs. Note that we use the definitions of a hybrid time domain, hybrid solution and complete and maximal solutions for systems described by (12) from [24, Ch. 2].

The following lemma shows the existence of complete solutions to (12). Furthermore, it provides a lower bound on the time between three consecutive switches, which suffices to show that no Zeno behavior occurs. We remind that $t_{j, i}$ denotes the time when the $i$ th switch of load $j$ occurs.

Lemma 1: For any initial condition $z(0,0) \in \Lambda$ there exists a complete solution to (12). Furthermore, for any complete bounded solution to (12), there exists $\tau>0$ such that $\min _{i \geq 1}\left(t_{j, i+2}-t_{j, i}\right) \geq \tau$ for any $j \in N$.

It should be noted that the condition for boundedness of solutions to (12) is not restrictive since it can be shown that all solutions to (12) are bounded. The latter, follows by noting that (12) consists of the asymptotically stable linear system (1), (2), in feedback with the hybrid system (4), (7) and that the magnitude of $d^{s}$, which can be regarded as the output of (4), (7), is bounded.

\section{Performance analysis}

In this section we state one of the main results of this paper, associated with the performance of solutions to (12). The following theorem demonstrates that as the number of loads tends to infinity, then for all initial conditions there exist arbitrarily long time intervals where frequency deviations are arbitrarily small.

Theorem 2: Consider the system described by (12) and let Assumption 1 and Design condition 1 hold. Furthermore, assume that the thermostatic loads described by (4), (7) satisfy $\bar{d}_{j}=\frac{\Gamma}{|N|}$. Then, as $|N| \rightarrow \infty$, for any $z(0,0) \in \mathbb{R}^{n} \times P^{|N|}$, any maximal solution of (12) and any $\epsilon>0, \hat{\tau} \in \mathbb{R}_{+}$, there exists $\tau \in \mathbb{R}_{+}$such that $|\omega(t, j)| \leq \epsilon$ for $t \in[\tau, \tau+\hat{\tau}]$.

The importance of Theorem 2 is that it shows, for all initial conditions, that frequency trajectories become arbitrarily small for an arbitrarily long amount of time. Also as shown in Lemma 1 the scheme in (7) avoids Zeno behaviour, and being deterministic, it allows the instant response to frequency deviations, thus providing improved ancillary services to the power system.

Preliminary analysis and numerical simulations indicate that a stronger version of Theorem 2 possibly holds, suggesting that, when the frequency control scheme (7) is implemented and the number of loads tends to infinity, frequency deviations are arbitrarily small with arbitrarily high probability (i.e. at times with arbitrarily high relative frequency of occurrence). However, the analytic derivation of the above statement raises various technical challenges and will be part of future work.

\section{CONCLUSION}

We have studied the problem of controlling thermostatic loads to provide ancillary services to the power network at urgencies. We first considered conventional thermostatic loads and showed that their aggregation has zero variance when their number tends to infinity and a mild condition on their period ratios holds. Then, we proposed a deterministic control scheme for thermostatic loads which induces switching when frequency deviations exceed particular frequency thresholds. For the considered scheme, we explain how frequency thresholds could be designed such that the coupling between load and frequency dynamics does not cause load synchronization. In particular, when the number of loads tends to infinity, we showed that frequency deviations are arbitrarily small for arbitrarily large periods of time.

\section{REFERENCES}

[1] H. Lund, "Large-scale integration of optimal combinations of pv, wind and wave power into the electricity supply," Renewable energy, vol. 31, no. 4, pp. 503-515, 2006.

[2] A. Ipakchi and F. Albuyeh, "Grid of the future," IEEE power and energy magazine, vol. 7, no. 2, pp. 52-62, 2009.

[3] A. Molina-Garcia, F. Bouffard, and D. S. Kirschen, "Decentralized demand-side contribution to primary frequency control," IEEE Transactions on Power Systems, 2011.

[4] A. Kasis, E. Devane, C. Spanias, and I. Lestas, "Primary frequency regulation with load-side participation part i: stability and optimality," IEEE Transactions on Power Systems, 2016.

[5] E. Devane, A. Kasis, M. Antoniou, and I. Lestas, "Primary frequency regulation with load-side participation-part ii: Beyond passivity approaches," IEEE Transactions on Power Systems, vol. 32, no. 5, pp. 3519-3528, 2016.

[6] A. Kasis, E. Devane, and I. Lestas, "Primary frequency regulation in power networks with ancillary service from load-side participation," IFAC-PapersOnLine, vol. 50, no. 1, pp. 4394-4399, 2017.

[7] A. Kasis, N. Monshizadeh, and I. Lestas, "Primary frequency regulation in power grids with on-off loads: chattering, limit cycles and convergence to optimality," arXiv preprint arXiv:1908.08077, 2019.

[8] E. Mallada, C. Zhao, and S. Low, "Optimal load-side control for frequency regulation in smart grids," in Communication, Control, and Computing (Allerton), 2014 52nd Annual Allerton Conference on, pp. 731-738, IEEE, 2014.

[9] A. Kasis, N. Monshizadeh, E. Devane, and I. Lestas, "Stability and optimality of distributed secondary frequency control schemes in power networks," IEEE Transactions on Smart Grid, 2017.

[10] S. Trip, M. Bürger, and C. De Persis, "An internal model approach to (optimal) frequency regulation in power grids with time-varying voltages," Automatica, vol. 64, pp. 240-253, 2016.

[11] A. Kasis, N. Monshizadeh, and I. Lestas, "A novel distributed secondary frequency control scheme for power networks with high order turbine governor dynamics," in 2018 European Control Conference (ECC), pp. 2569-2574, IEEE, 2018.

[12] S. Trip and C. De Persis, "Distributed optimal load frequency control with non-passive dynamics," IEEE Transactions on Control of Network Systems, vol. 5, no. 3, pp. 1232-1244, 2017.

[13] A. Kasis, N. Monshizadeh, and I. Lestas, "Secondary frequency control with on-off load side participation in power networks," IEEE Transactions on Control of Network Systems, 2019.

[14] J.-P. Zimmermann, M. Evans, J. Griggs, N. King, L. Harding, P. Roberts, and C. Evans, "Household electricity survey: A study of domestic electrical product usage," Intertek Testing \& Certification Ltd, 2012.

[15] J. A. Short, D. G. Infield, and L. L. Freris, "Stabilization of grid frequency through dynamic demand control," IEEE Transactions on Power Systems, 2007.

[16] D. Angeli and P.-A. Kountouriotis, "A stochastic approach to "dynamic-demand" refrigerator control," IEEE Transactions on control systems technology, vol. 20, no. 3, pp. 581-592, 2012.

[17] M. Aunedi, P.-A. Kountouriotis, J. O. Calderon, D. Angeli, and G. Strbac, "Economic and environmental benefits of dynamic demand in providing frequency regulation," IEEE Transactions on Smart Grid, vol. 4, no. 4, pp. 2036-2048, 2013.

[18] S. H. Tindemans, V. Trovato, and G. Strbac, "Decentralized control of thermostatic loads for flexible demand response," IEEE Transactions on Control Systems Technology, vol. 23, no. 5, pp. 1685-1700, 2015. 
[19] L. C. Totu, R. Wisniewski, and J. Leth, "Demand response of a tcl population using switching-rate actuation," IEEE Transactions on Control Systems Technology, vol. 25, no. 5, pp. 1537-1551, 2017

[20] A. R. Bergen and V. Vittal, Power Systems Analysis. Prentice Hall, 1999.

[21] P. M. Anderson and M. Mirheydar, "A low-order system frequency response model," IEEE Transactions on Power Systems, vol. 5, no. 3, pp. 720-729, 1990.

[22] Q. Shi, F. Li, and H. Cui, "Analytical method to aggregate multimachine sfr model with applications in power system dynamic stud- ies," IEEE Transactions on Power Systems, vol. 33, no. 6, pp. 63556367, 2018.

[23] M. Stadler, W. Krause, M. Sonnenschein, and U. Vogel, "Modelling and evaluation of control schemes for enhancing load shift of electricity demand for cooling devices," Environmental Modelling \& Software, vol. 24, no. 2, pp. 285-295, 2009.

[24] R. Goebel, R. G. Sanfelice, and A. R. Teel, Hybrid Dynamical Systems: modeling, stability, and robustness. Princeton University Press, 2012 